\title{
Investigating the expression, effect and tumorigenic pathway of PADI2 in tumors
}

\author{
This article was published in the following Dove Press journal: \\ OncoTargets and Therapy \\ 8 March 2017 \\ Number of times this article has been viewed
}

\author{
Wei Guo ${ }^{1,2, *}$ \\ Yabing Zheng ${ }^{2, *}$ \\ Bing $X u^{\prime}$ \\ Fang $\mathrm{Ma}^{\prime}$ \\ Chang $\mathrm{Li}^{3}$ \\ Xiaoqian Zhang ${ }^{4}$ \\ Yao Wang' \\ Xiaotian Chang' \\ 'Medical Research Center, Shandong \\ Provincial Qianfoshan Hospital, \\ 2Obstetrical Department, Shandong \\ Provincial Qianfoshan Hospital, \\ Shandong University, Jinan, Shandong, \\ ${ }^{3}$ Pathology Department, Tengzhou \\ Central People's Hospital, Tengzhou, \\ ${ }^{4}$ Clinical Laboratory, PKU Care \\ Luzhong Hospital, Zibo, Shandong, \\ People's Republic of China \\ *These authors contributed equally \\ to this work
}

Background: Peptidylarginine deiminase (PAD) catalyzes the conversion of arginine residues to citrulline residues, termed citrullination. Recent studies have suggested that PAD isoform 2 (PADI2) plays an important role in tumors, although its tumorigenic effect and mechanism are largely unknown.

Materials and methods: Immunohistochemistry and enzyme-linked immunosorbent assay (ELISA) were used to investigate the expression level of PADI2 in various tumor tissues and patient blood samples, respectively. MNK-45 and Bel-7402 tumor cell lines originating from gastric and liver tumors, respectively, were treated with anti-PADI2 siRNA, and the subsequent cell proliferation, apoptosis and migration were observed. Polymerase chain reaction (PCR) arrays, including Cancer PathwayFinder, Oncogenes and Tumor Suppressor Genes, p53 Signaling Pathway, Signal Transduction Pathway and Tumor Metastasis PCR arrays, were used to investigate the tumorigenic pathway of PADI2 in the siRNA-treated tumor cells. This analysis was verified by real-time PCR.

Results: Immunohistochemistry detected significantly increased expression of PADI2 in invasive breast ductal carcinoma, cervical squamous cell carcinoma, colon adenocarcinoma, liver hepatocellular carcinoma, lung cancer, ovarian serous papillary adenocarcinoma and papillary thyroid carcinoma samples. ELISA detected a twofold increase in PADI2 expression in the blood of $48.3 \%$ of patients with liver cancer, $38 \%$ of patients with cervical carcinoma and $32 \%$ of patients with gastric carcinoma. Increased apoptosis and decreased cell proliferation and migration were observed in the anti-PADI2 siRNA-treated MNK-45 cells, and increased cell proliferation and migration and decreased apoptosis were observed in the treated Bel-7402 cells with suppressed PADI2 expression. PCR arrays and real-time PCR detected significantly decreased CXCR2 and EPO expression in the MNK-45 cells and Bel-7402 cells, respectively, with the anti-PADI2 siRNA treatments.

Conclusion: PADI2 expression is increased in many types of tumor tissues and patient blood samples. PADI2 may advance abnormal cell behavior in gastric cancers by mediating CXCR2, a well-known gene that stimulates cell proliferation and invasion. However, PADI2 might have deleterious effects on tumor growth and metastasis in liver tumor cells by regulating the expression of EPO, a gene with controversial functions in tumor growth. The results suggest that the effect of PADI2 on tumorigenesis is multifactorial, depending on the tumor type.

Keywords: PADI2, tumorigenesis, pathway, CXCR2, EPO

\section{Introduction}

Peptidylarginine deiminase (PAD) conducts the post-translational modification of arginine residues to citrulline residues, referred to as citrullination or deimination. Five mammalian PAD family members (PAD or PADI 1-4 and 6) are all encoded by a cluster of genes on chromosome 1p36.13 and have a described tissue-specific distribution in most body tissues. ${ }^{1}$ PAD-mediated post-translational citrullination is
Correspondence: Xiaotian Chang

Qianfoshan Hospital, Shandong

University, Jingshi Road 16766,

Jinan, Shandong 2500I4, People's

Republic of China

Tel +8653189268970

Email changxt@।26.com
OncoTargets and Therapy 20।7:10 |475-|485

1475

Dovepress f in 0

http://dx.doi.org/10.2147/0TT.S92389 
involved in various physiological and pathogenic activities by altering the structural stability of target proteins., ${ }^{2,3}$

The pathological roles of the PAD family and citrullination in tumors have attracted increased interest. ${ }^{4-6}$ PAD isoform 2 (PAD2/PADI2), a member of the PAD family, has been implicated in cancer. McElwee et $\mathrm{al}^{7}$ identified PADI2 as a potential breast cancer biomarker and therapeutic target and also found that PADI2-overexpressing transgenic mice were susceptible to spontaneous skin neoplasia. ${ }^{8}$ Tanday $^{9}$ recently reported that inhibiting the expression of PADI2 could halt the progression of myeloma. We recently reported that PADI2 is a gene susceptible to breast cancer risk, and its expression contributes to tumorigenesis via ACSL4, BINC3 and CA9 signaling. ${ }^{10}$ These findings suggest that PADI2 is involved in the tumorigenesis of many tumors and plays an important role in progression. Alternatively, Cantarino et $\mathrm{al}^{11}$ demonstrated that downregulation of PADI2 is an early event in colorectal carcinogenesis. Therefore, the expression of PADI2 in various tumor types and blood of patients requires a thorough investigation. The effect of PADI2 on the tumorigenic process and regulatory mechanism should be comparatively studied among different tumors.

To gain additional insight into the tumorigenic role of PADI2, the present study analyzed the expression level of PADI2 in various tumor tissues and blood of patients. Based on these observations, we investigated the effects of PADI2 on cultured gastric and hepatic tumor cells. We also used a polymerase chain reaction (PCR) array to determine the tumorigenic signaling pathway of PADI2 in these two cell lines. Our aim was to understand the role of PADI 2 in the tumorigenic process and compare the regulatory pathways in different tumor cell lines.

\section{Materials and methods Immunohistochemistry}

Tissue arrays (Alenabio, Xian, People's Republic of China) were used for immunohistochemistry to determine PADI2 expression. These slides contained tumor and normal samples from each of the following tissues: breast (35 invasive ductal carcinoma and 8 normal tissue samples), cervical (52 squamous cell carcinoma and 10 normal tissue samples), colon (36 adenocarcinoma and 8 normal tissue samples), esophageal (54 squamous cell carcinoma and 8 normal tissue samples), liver (38 hepatocellular carcinoma and 8 normal tissue samples), lung (27 squamous cell carcinoma and 5 normal tissue samples), ovarian (40 serous papillary adenocarcinoma and 10 normal tissue samples), rectal (70 adenocarcinoma and 10 normal rectal tissue samples), stomach (70 adenocarcinoma and 10 normal tissue samples) and thyroid carcinoma (60 papillary carcinoma and 10 normal tissue samples). The manufacturer supplied the clinical data for each participant, including age, sex, clinical pathological diagnosis and origin (Table S1). The tissue sections were deparaffinized and rehydrated using standard procedures. The tissue sections were heated at $95^{\circ} \mathrm{C}$ for $10 \mathrm{~min}$ in citrate buffer solution (Sigma-Aldrich, Shanghai, People's Republic of China) for antigen recovery and were incubated with an endogenous peroxidase inhibitor (MaixinBio, China) for $30 \mathrm{~min}$ at room temperature. After washing with phosphate buffered saline (PBS), the sections were incubated overnight with anti-PADI2 antibody (Abcam, Cambridge, MA, USA) at $4^{\circ} \mathrm{C}$. The PADI2 antibody was raised against a KLH-conjugated synthetic peptide derived from within residues 100-200 of human PADI2. The specificity and cross-reactivity of the antibody were verified by the manufacturer using human tissues and were found to be suitable for Western blotting and immunohistochemistry. The immunosignal was displayed using the UltraSensitive TM S-P Kit (Maixin-Bio, Beijing, People's Republic of China) according to the manufacturer's instructions. The 3,3'-Diaminobenzidine tetrahydrochloride substrate revealed immunoreaction in yellow color. The specificity and optimized antibody dilution of the anti-PADI2 antibody were determined by incubating the slides with goat preimmune serum (Maixin-Bio) or modification buffer without the addition of antibody.

The expression level of PADI2 in tumor tissue sections was semiquantified using the Chiew-Loon Koo's modified semiquantitative scoring system, ${ }^{12}$ which has been widely accepted and used in other studies. ${ }^{12}$ This system considers the staining intensity and extent of the area stained. Every tumor section was given a score according to the intensity of the nucleic or cytoplasmic staining (no staining $=0$, weak staining $=1$, moderate staining $=2$ and strong staining $=3$ ) and the extent of stained cells $(0 \%=0,1 \%-10 \%=1,11 \%-$ $50 \%=2,51 \%-80 \%=3,81 \%-100 \%=4$; negative means $0 \%$ area staining, focally positive means $1 \%-80 \%$ area staining and diffusely positive means $81 \%-100 \%$ area staining). The final immunoreactive scores were obtained by multiplying the intensity scores with the extent of positivity scores of the stained tissues, for which the minimum score is 0 , and the maximum score is 12 .

All the experiments in the present study were examined and approved by the Ethics Committee of Shandong Provincial Qianfoshan Hospital, who also approved the study. All patients provided written informed consent to participate in the study. 


\section{Enzyme-linked immunosorbent assay (ELISA)}

Peripheral blood samples were collected from patients with breast cancer $(\mathrm{n}=259$, age $=26-79$ years, mean age $=49$ years $)$, cervical cancer $(n=216$, age $=23-88$ years, meanage $=50.9$ years , esophageal cancer $(\mathrm{n}=83,8$ females, age $=38-76$ years, mean age $=61$ years $)$, gastric cancer $(n=120,30$ females, age $=28-80$ years, mean age $=58$ years), liver cancer $(\mathrm{n}=178,35$ females, age $=29-83$ years, mean age $=57$ years $)$, lung cancer $(\mathrm{n}=128,50$ females, age $=34-85$ years, mean age $=57$ years $)$, ovarian cancer $(n=128$, age $=13-82$ years, mean age $=55$ years $)$ or rectal cancer $(n=116,37$ females, age $=25-85$ years, mean age $=56$ years). Four hundred sixtyfive ( 202 females, age $=17-59$ years) healthy individuals with a mean age of 32 years were blood donors. Following centrifugation at $1,000 \times \mathrm{g}$ for $30 \mathrm{~min}$, serum samples were collected and stored at $-80^{\circ} \mathrm{C}$ prior to use. Blood samples were diluted 10 -fold and incubated in plates for $2 \mathrm{~h}$ at $37^{\circ} \mathrm{C}$. The plates were washed three times with PBS Tween-20 (PBST), and the wells were incubated for $1 \mathrm{~h}$ at $37^{\circ} \mathrm{C}$ with $0.5 \%$ bovine serum albumin. Following an additional wash with PBST, the anti-PADI2 antibody (LSBio), diluted 3,000fold, was added to the plates, which were incubated for $1 \mathrm{~h}$ at $37^{\circ} \mathrm{C}$. The antibody was raised against synthetic peptide derived from the $\mathrm{C}$-term domain of the human PADI2 protein. The manufacturer confirmed that the antibody reacts with the $76 \mathrm{kDa}$ PADI2 protein from human, mouse and rat and has no cross-reaction with the other PAD isoforms. The antibody was found to be suitable for Western blotting, immunoprecipitation and ELISA. Following a washing step, Peroxidase Affinipure Goat anti-rabbit IgG (Jackson Immunoresearch, West Grove, PA, USA), diluted 4,000-fold, was added, and the plates were incubated for $30 \mathrm{~min}$ at $37^{\circ} \mathrm{C}$. Following another PBST wash, the plates were developed by adding a 3,3',5,5'-Tetramethylbenzidine liquid substrate for ELISA (Sigma). The absorbance at $450 \mathrm{~nm}$ was measured using a plate reader (Thermo Scientific, Waltham, MA, USA). The serum level of PADI2 was determined according to Briggs's $P / N$ method. ${ }^{13}$ The optical density $(\mathrm{OD})_{450}$ value of the patient serum ( $P$-value) was divided by the $\mathrm{OD}_{405}$ value of the pooled healthy sera ( $N$ value). The $P / N$ ratio was calculated for each serum sample.

\section{Cell culture and siRNA interference}

MNK-45 and Bel-7402 cell lines that were originated from liver cancer and gastric cancer, respectively, were cultured in an atmosphere of $5 \% \mathrm{CO}_{2}$ at $37^{\circ} \mathrm{C}$. Dulbecco's Modified Eagle's Medium (DMEM) contained 10\% fetal calf serum,
$50 \mathrm{U} / \mathrm{mL}$ penicillin and $50 \mu \mathrm{g} / \mathrm{mL}$ streptomycin. The siRNA oligonucleotides targeting the PADI2 gene (target sequence: 5'-CCCGTTCTTCGGCCAACGCTA-3') were commercially obtained from Qiagen (Duesseldorf, Germany). MNK-45 and Bel-7402 tumor cells were transfected with the anti-PADI2 siRNAs at $20 \mathrm{nM}$ using the HiPerFect transfection reagent (Qiagen). Transfection was conducted for $48 \mathrm{~h}$. The inhibition of PADI2 expression in these cell lines was verified using real-time PCR. Transfection with Mm/Hs-MAPK1 siRNA (AATGCTGACTCCAAAGCTCTG) that specifically suppresses MAPK1 expression was prepared as positive controls, and transfection with Allstar siRNA that has no effect on any gene expression was used as negative controls.

\section{Cell proliferation assay}

MNK-45 and Bel-7402 cells were cultured onto 96-well culture plates until they grew to $80 \%$ confluence. AntiPADI2 siRNA was added to the culture, and the cells were transfected in culture medium for $24-72 \mathrm{~h}$ at $37^{\circ} \mathrm{C}$ in $5 \%$ $\mathrm{CO}_{2}$. A total of $10 \mu \mathrm{L}$ of cell counting kit-8 solution (CCK-8; Dojindo, Kumamoto, Japan) was added to each well, and the cells were continually incubated for an additional $4 \mathrm{~h}$. The absorbance was measured at $450 \mathrm{~nm}$ using a spectrophotometer (Spectramax 190; Molecular Devices, Sunnyvale, CA, USA).

\section{Cell apoptosis assay}

MNK-45 cells or Bel-7402 cells with the treatment of anti-PADI2 siRNA were washed twice with PBS and were resuspended in binding buffer at a particular concentration. The cell suspensions at a concentration of $1 \times 10^{5}$ cells $/ 100 \mu \mathrm{L}$ were transferred into $5 \mathrm{~mL}$ culture tubes, and $5 \mu \mathrm{L}$ of annexin V-phycoerythrin (eBioscience, San Diego, CA, USA) and $5 \mu \mathrm{L}$ of 7-amino-actinomycin (eBioscience) were added to the tube. The mixture was placed at room temperature in the dark for $15 \mathrm{~min}$. Later, another $400 \mu \mathrm{L}$ aliquot of binding buffer was added. The apoptosis ratio was examined by flow cytometry (FACSAria II; BD Biosciences, Franklin Lakes, NJ, USA).

\section{Transwell migration assay}

The cell migration assay of MNK-45 cells or Bel-7402 cells with the treatment of anti-PADI2 siRNA was performed using Transwell inserts $\left(8 \mu \mathrm{m}\right.$ pore size; $\left.\operatorname{Costar}^{\circledR}\right)$. The transfected cells at a concentration of $5 \times 10^{4}$ cells $/ 200 \mu \mathrm{L}$ were suspended in serum-free media and were added to the upper chamber, and $500 \mu \mathrm{L}$ of complete DMEM was added to the lower chamber of Transwell inserts. The cells were 
incubated for $24 \mathrm{~h}$. The filter of the chambers was immersed in methanol for $15 \mathrm{~min}$ at room temperature, treated with $0.25 \%$ crystal violet stain for $10 \mathrm{~min}$ at room temperature and washed with water. The cells that had migrated to the lower side of the filter were observed and counted using an inverted fluorescence microscope.

\section{PCR array analysis}

Bel-7402 and MNK45 cells were transfected with anti-PADI2 siRNA as described earlier. Total RNA was isolated from the treated cells using Trizol solution (Invitrogen, Carlsbad, CA, USA) according to the manufacturer's protocol. RNA samples were reversed transcribed into first-strand cDNA using the $\mathrm{RT}^{2}$ First Strand Kit. The Cancer PathwayFinder, Oncogenes and Tumor Suppressor Genes, p53 Signaling Pathway, Signal Transduction Pathway and Tumor Metastasis PCR arrays (Qiagen) were used to examine significantly changed transcriptions of tumor-related genes in Bel-7402 and MNK-45 cells with inhibited PADI4 expression. The PCR array analysis was conducted using the ViiA7 DX system (Life Science, Waltham, MA, USA) according to the manufacturer's instructions. The cDNA was mixed with an appropriate $\mathrm{RT}^{2} \mathrm{SYBR}$ Green Mastermix, and this mixture was aliquoted into the wells of the $\mathrm{RT}^{2}$ Profiler PCR Array. The relative expression levels of the target genes were determined using the data from the real-time cycler and $\Delta \Delta \mathrm{CT}$ method. The raw array data were analyzed by the PCR Array Data Analysis System at http://sabiosciences. com/pcrarraydataanalysis.php. Fold changes were expressed as log-normalized ratios of the target gene expression in anti-PADI2 siRNA-treated cells/expression in Allstar siRNAtreated controls. Fourfold or higher changes in expression were considered to be biologically significant in the study according to the instructions of the manufacturer.

\section{Real-time PCR}

Total RNA was extracted from MNK-45 or Bel-7402 and was reverse transcribed to first-strand cDNA as described earlier. The PCR amplification was conducted in a total volume of $10 \mu \mathrm{L}$, containing $1 \mu \mathrm{L}$ of cDNA, $5 \mu \mathrm{L}$ of SYBR Green Real-time PCR Master Mix (Toyobo, Osaka, Japan) and $1 \mu \mathrm{L}$ of each primer. Real-time PCR was performed using the ViiA7 DX system. The reaction condition was set according to the annealing temperature of the primers. Two reactions were simultaneously performed in each sample: one reaction was performed to detect the mRNA level of the target gene and the other to detect the level of $\beta$-actin. PCR products were confirmed by melting curve analysis. The relative mRNA level was calculated using the comparative threshold cycle $(\mathrm{Ct})$ method. The target gene expression level was normalized to the $\beta$-actin mRNA expression level. Forward primer and reverse primer sequences for real-time PCR were obtained from Qiagen and were designed as follows: PADI2: 5' ${ }^{\prime}$ TGAAAGAGGTGAAGAACCTTG-3' and 5'-GTTTAGGTACTGGAAGCAGAC-3'; $\beta$-actin: 5'-TGG CACCCAGCACAATGAA-3' and 5'-CTAAGTCA TAGTCCGCCTAGAAGCA-3'; CXCR2: 5'-CCC ATGTGAACCAGAATCC-3' and 5'-GAATACC AGGGCATAGATAATGAC-3'; EPO: 5'-AGCTT GAATGAGAATATCACTGT-3' and 5'-CCTCTTCCA GGCATAGAAA-3'.

\section{Statistical analysis}

Two-tailed Student's $t$-test was used to analyze experimental data. A value of $P<0.05$ was considered a statistically significant difference. All the experiments in the study were performed three times or more to verify the results.

\section{Results Detection of PADI2 expression in tumor tissues}

Immunohistochemistry was performed to locate PADI2 expression in a panel of tumor tissues. Significant expression of PADI2 was observed in $95 \%$ of breast-invasive ductal carcinomas, $34.1 \%$ of cervical squamous cell carcinomas, $97.5 \%$ of colon adenocarcinomas, $88 \%$ of esophageal cancers, $62.8 \%$ of gastric adenocarcinomas, $55.2 \%$ of liver hepatocellular carcinomas, $92.5 \%$ of lung cancers, $30 \%$ of ovarian serous papillary adenocarcinomas, $58.5 \%$ of rectal adenocarcinomas and $90 \%$ of papillary thyroid carcinoma samples. The immunosignal was localized to the cytoplasm of the tumor cells. PADI2 was not detected in normal cervical tissue, lung tissue, ovarian tissue or thyroid tissue, except in a few mesenchymal-like cells and certain endothelial cells. PADI2 was also localized to selected epidermis regions of normal breast tissue, colon tissue, esophageal tissue, gastric tissue, liver tissue and rectal tissue, but the density of the immunosignal was relatively lower. The immunohistochemical observation is shown in Figure 1. In addition, the immunoreactive score analysis indicated a significantly increased expression of PADI2 in breast-invasive ductal carcinomas, cervical squamous cell carcinomas, colon adenocarcinomas, liver hepatocellular carcinomas, lung cancers, ovarian serous papillary adenocarcinomas and papillary thyroid carcinoma samples compared with the corresponding normal tissues $(P<0.001)$. The analytical result is also shown in Figure 2.

Sandwich ELISA was used to determine the level of PADI2 in the blood of patients with tumors. The PADI2 level in the 


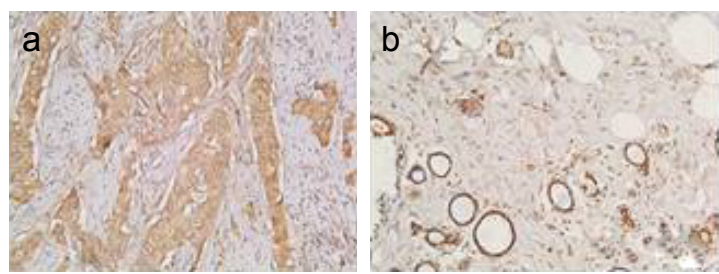

Breast tissues

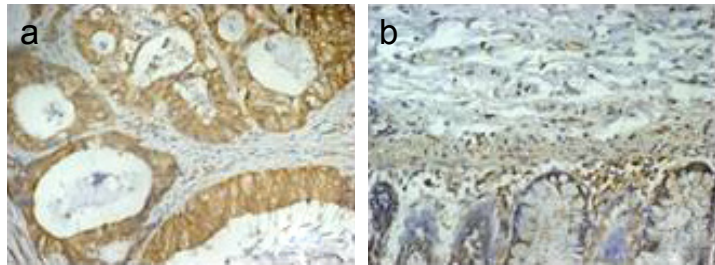

Colonal tissues

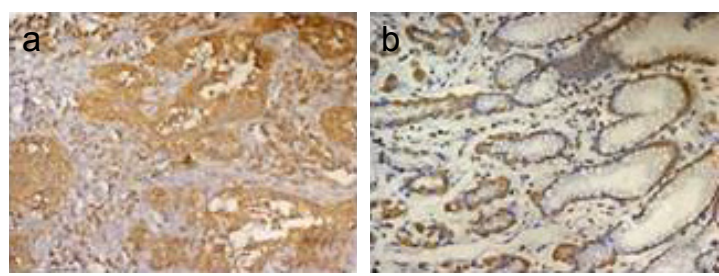

Gastric tissues

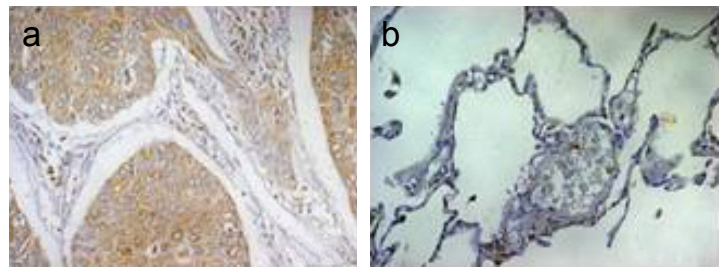

Lung tissues

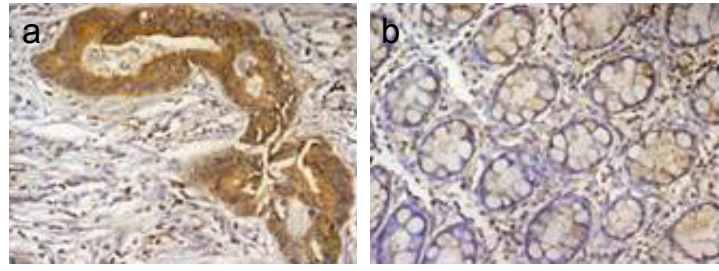

Rectal tissues
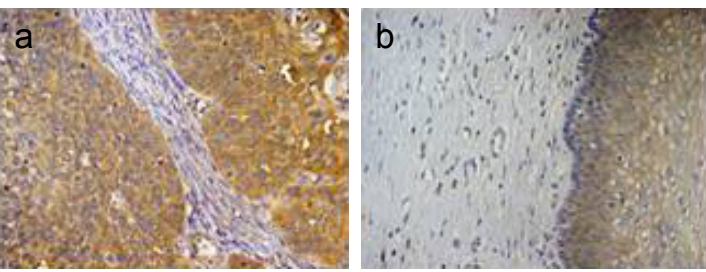

Cervical tissues

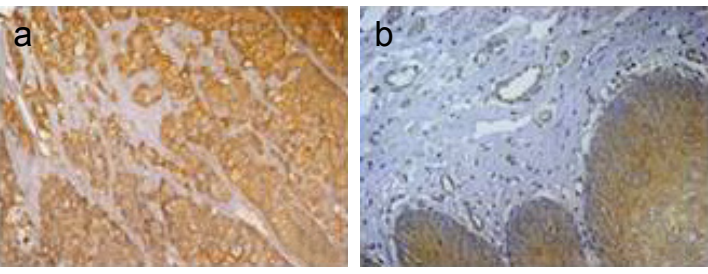

Esophageal tissues

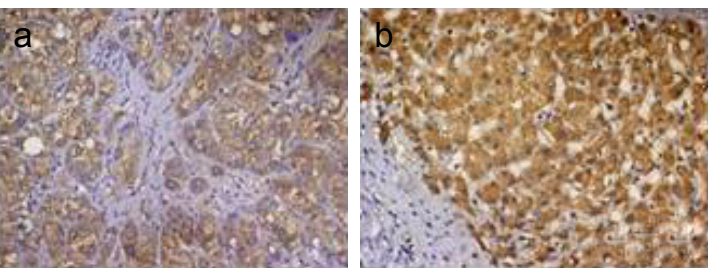

Liver tissues
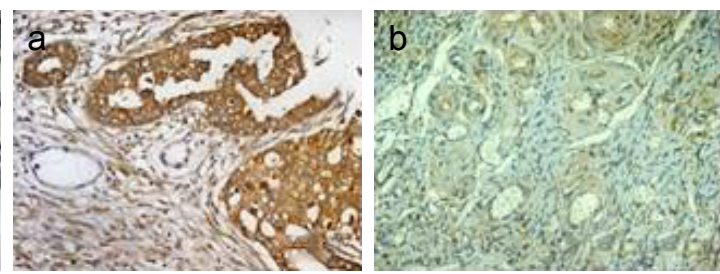

Ovarian tissues

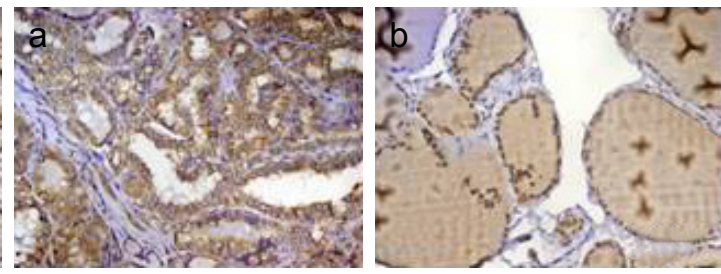

Thyroid tissues

Figure I Immunodetection of PADI2 in various tumor tissues.

Notes: PADI2 shows significantly increased expression in many tumor types. "a" represents tumor tissue, and "b" represents the parallel tumor adjacent tissues or normal tissues. Original magnification: $200 \times$.

Abbreviation: PADI2, peptidylarginine deiminase isoform 2.

pooled blood of healthy subjects was used as the standard. $P / N$ ratios $\geq 2.1$ were considered positive in this study. PADI2 showed positive expression in the blood of $48.3 \%$ of patients with liver cancer, $38 \%$ of patients with cervical carcinoma and $32 \%$ of patients with gastric carcinoma. The enzyme also showed positive expression in $20 \%-30 \%$ of patients with other tumors. The ELISA results are shown in Figure 3.

The immunohistochemical observations, immunoreactive score analysis and ELISA results suggest that PADI2 has significantly increased expression in many types of tumor tissues and peripheral blood of patients, especially patients with breast cancer, gastric cancer or liver cancer.
Detection of cell proliferation, apoptosis and migration of MNK-45 and Bel-7402 cells treated with anti-PADI2 siRNA

MNK-45 and Bel-7402 cells were treated with anti-PADI2 siRNA as described earlier. Real-time assays detected significantly decreased transcription of PADI2 in the anti-PADI2 siRNA-treated cells (Figure S1). The CCK-8 assay was used to measure the cell proliferation. The analysis showed a significantly declined cell proliferation in the siRNA-treated MNK-45 cells compared with cells treated with the Allstar siRNA $\left(P=1.71 \times 10^{-4}\right)$. Meanwhile, the CCK- 8 assay detected a significant increase in the cell proliferation of Bel-7402 


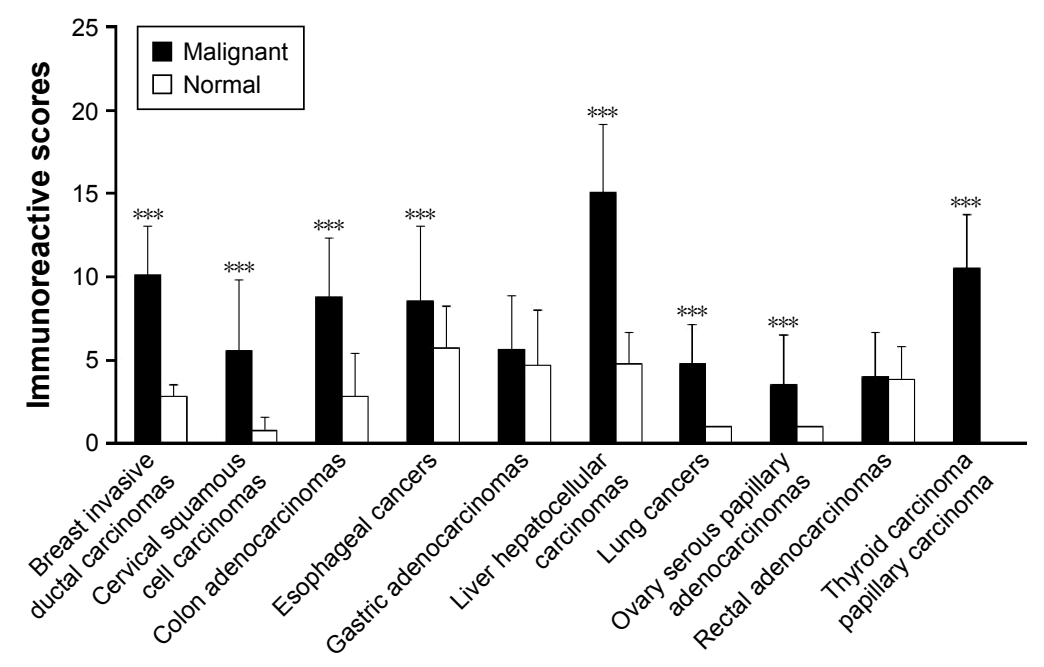

Figure 2 Semiquantitation of the PADI2 expression level in various tumor tissues.

Notes: The $x$-axis indicates tumor types and matched normal tissues, and the $y$-axis indicates the expression level of PADI2. $* * * P<0.001$. Abbreviation: PADI2, peptidylarginine deiminase isoform 2.

cells with the treatment of the anti-PADI2 siRNA compared with controls $(P=0.04)$. The result is shown in Figure 4.

Flow cytometric analysis was used to measure apoptosis in MNK-45 and Bel-7402 cells with the decreased PADI2 expression. Compared with the Allstar siRNA-treated cells, the annexin V/PE and 7-AAD double staining detected significantly increased apoptosis in the anti-PADI2 siRNAtreated MNK-45 cells $\left(P=4.98 \times 10^{-5}\right)$, but the analysis measured a considerably decreased apoptosis in the siRNA-treated Bel-7402 cells $\left(P=3 \times 10^{-4}\right)$. The result is shown in Figure 5 .

A two-compartment transwell system was used to measure cell migration in MNK-45 and Bel-7402 cells. The analysis detected a significantly decreased migration of MNK- 45 cells when the PADI2 expression was suppressed by anti-PADI2
siRNA compared with the cells treated with Allstar siRNA $\left(P=6.03 \times 10^{-5}\right)$; meanwhile, the assay observed a significantly increased cell migration in the siRNA-treated Bel-7402 cells $\left(P=3.6 \times 10^{-4}\right)$. The result is shown in Figure 6.

\section{Determination of the pathogenic pathway of PADI 2 in tumorigenesis}

A series of PCR arrays was used to determine the signaling pathway of PADI2 in tumorigenesis. MNK-45 cells and Bel-7402 cells were treated with anti-PADI2 siRNA. Real-time PCR detected a significant inhibition of the siRNA on PADI2 expression compared with the cells treated with Allstar siRNA. The result is shown in Figure S1. The Cancer PathwayFinder PCR array detected significantly decreased

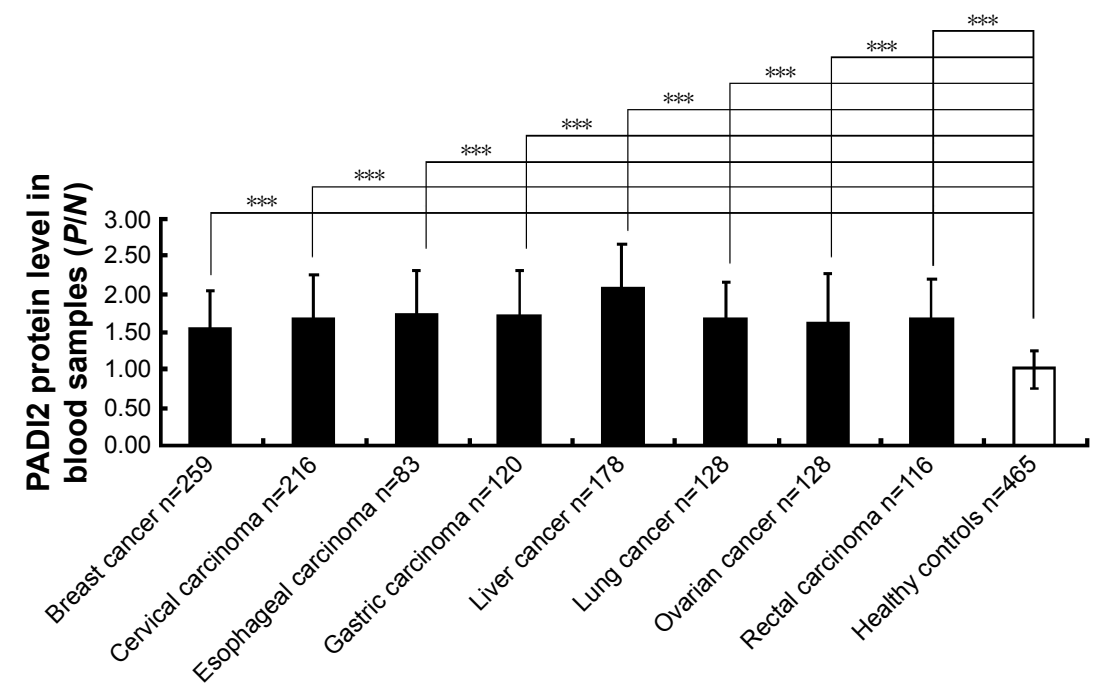

Figure 3 Determination of PADI2 levels in the blood samples of patients with tumors using ELISA.

Notes: The $x$-axis represents various tumor types, and the $y$-axis represents the PADI2 expression level with $P / N$ values. The $O D_{450}$ value of the positive serum ( $P$-value) was divided by the $O D_{405}$ value of the pooled negative sera $(N$ value). The $P / N$ ratio was calculated for each serum sample. $* * * P<0.001$.

Abbreviations: PADI2, peptidylarginine deiminase isoform 2; ELISA, enzyme-linked immunosorbent assay; OD, optical density. 
A

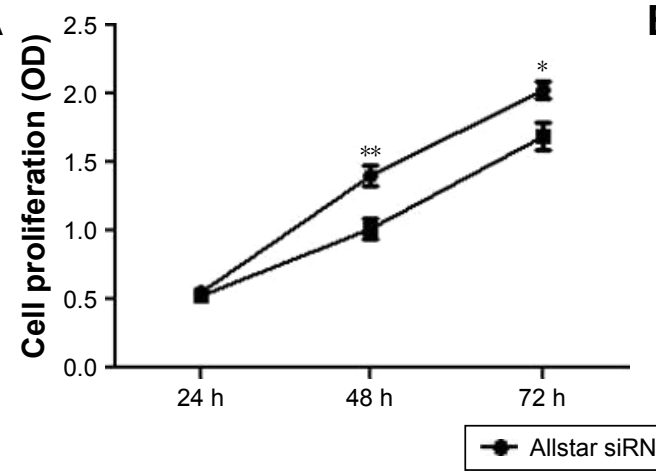

B

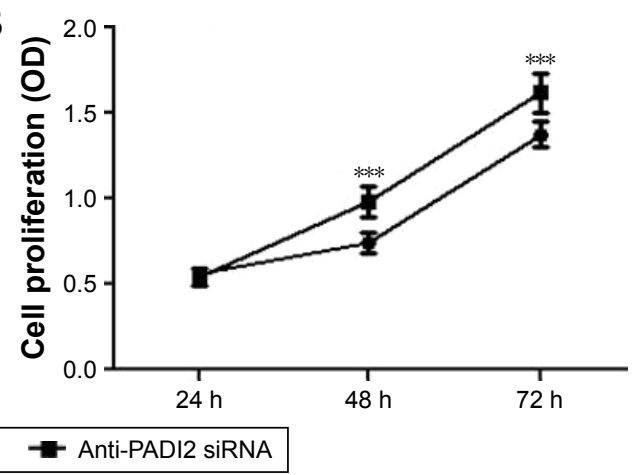

Figure 4 Proliferation of MNK-45 cells and Bel-7402 cells treated with anti-PADI2 siRNA.

Notes: (A) The CCK-8 assay detected MNK-45 viable cell numbers as represented by an OD value at $405 \mathrm{~nm}$. (B) The CCK-8 assay detected Bel-7402 viable cell numbers as represented by an OD value at $405 \mathrm{~nm}$. Cells treated with Allstar siRNA were used as negative controls. $* P<0.05, * * P<0.01$ and $* * * P<0.001$.

Abbreviations: PADI2, peptidylarginine deiminase isoform 2; CCK-8, cell counting kit-8; OD, optical density.

CA9 and IGFBP3 expression in the MNK-45 cells and decreased TEK expression in the Bel-7402 cells after these two cell lines were treated with anti-PADI2 siRNA. The Oncogenes and Tumor Suppressor Genes PCR array detected significantly decreased ESR 1 expression in the MNK-45 cells. The p53 Signaling PCR array detected significantly increased expression of TP63 in MNK-45 cells. The Signal Transduction PCR array detected significantly increased ACSL5, AXIN2, CCL5, HMOX1 and MMP7 expression and decreased CA9 expression in the MNK-45 cells, as well as decreased EPO expression in the Bel-7402 cells. The Tumor Metastasis PCR array detected significantly increased expression levels of MMP2 and MMP7 and decreased CXCR2 expression levels in MNK-45 cells. The results are shown in Figure 7, and the raw data are presented in Figure S2.

Those genes with significantly changed expression that were detected using PCR arrays were verified using real-time PCR. The real-time PCR results confirmed the 7.25-fold decrease in CXCR2 expression in MNK-45 cells and the 5.8-fold decrease in EPO in Bel-7402 cells following
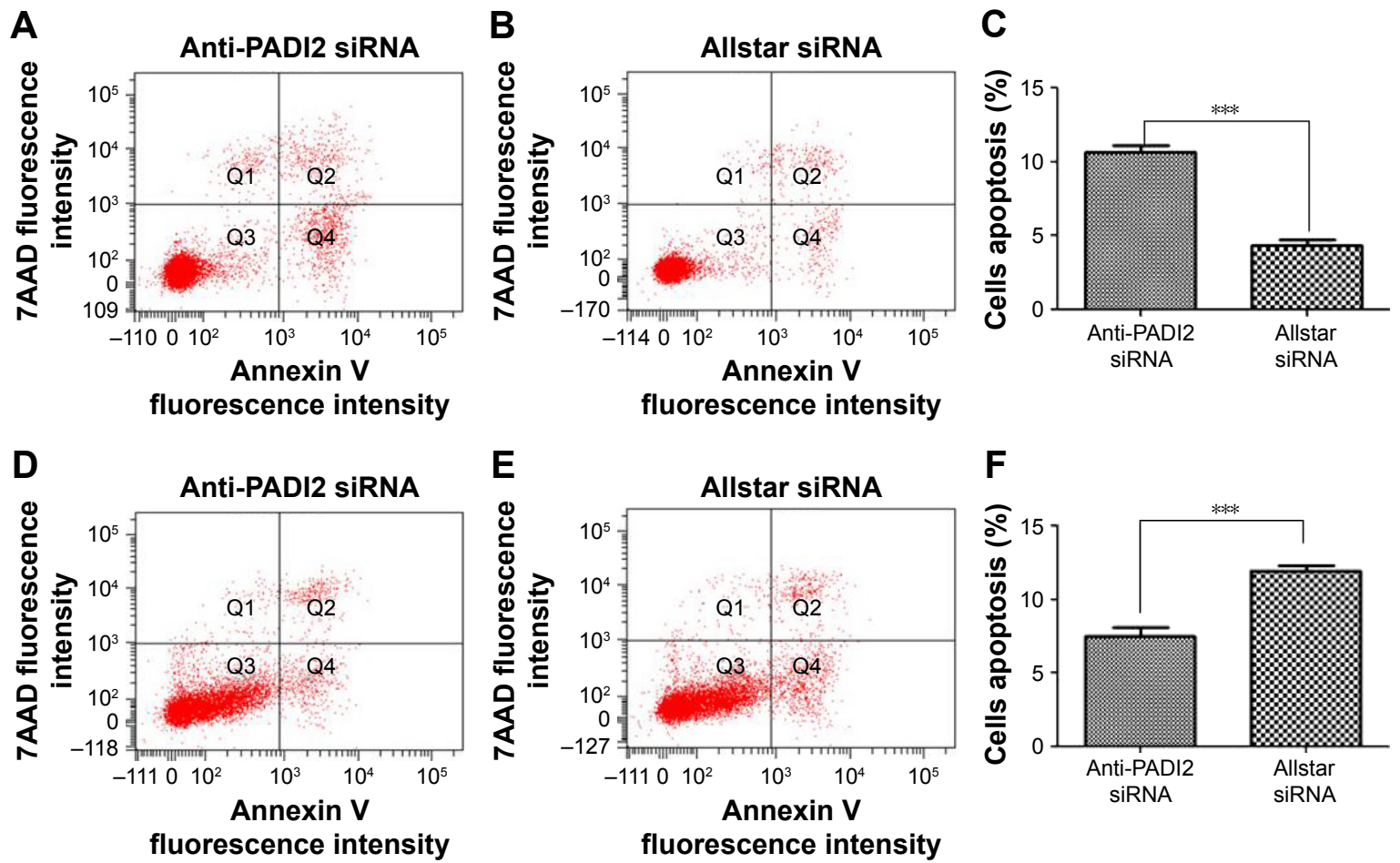

Figure 5 Apoptosis in MNK-45 cells and Bel-7402 cells treated with anti-PADI2 siRNA, as measured using the annexin $V$ cell apoptosis assay.

Notes: (A) MNK-45 cells were treated with anti-PADI2 siRNA. (B) MNK-45 cells treated with Allstar siRNA were used as negative controls. (C) Result of the apoptosis assay for MNK-45 cells. (D) Bel-7402 cells were treated with anti-PADI2 siRNA. (E) Bel-7402 cells treated with Allstar siRNA were used as negative controls. (F) Result of the apoptosis assay for MNK-45 cells. $* * * P<0.05$.

Abbreviation: PADI2, peptidylarginine deiminase isoform 2. 


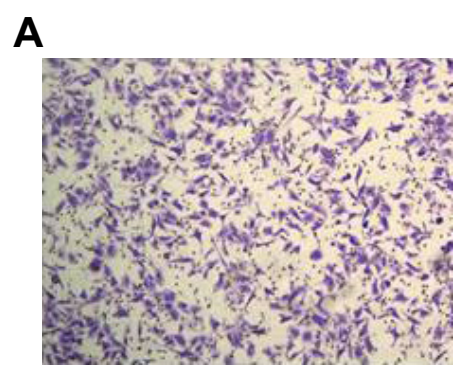

Anti-PADI2 siRNA

D

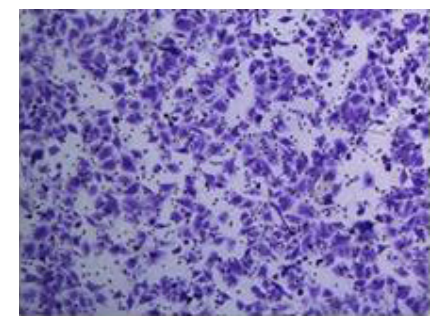

Anti-PADI2 siRNA
B

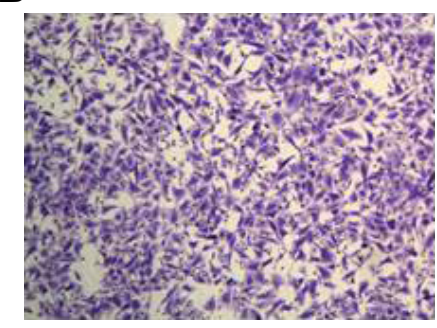

Allstar siRNA

E

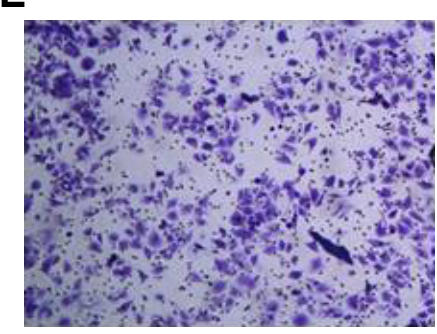

Allstar siRNA

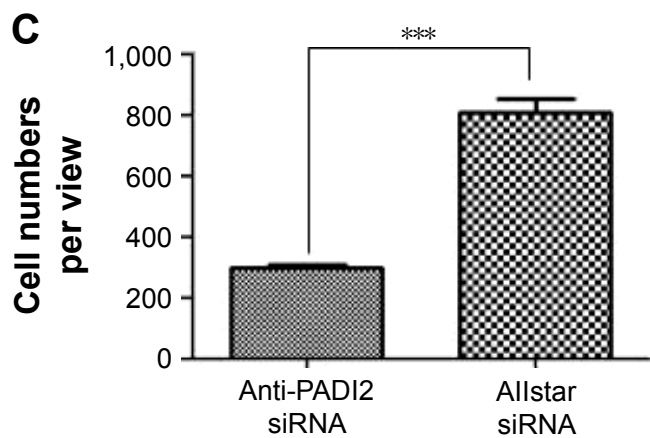

F

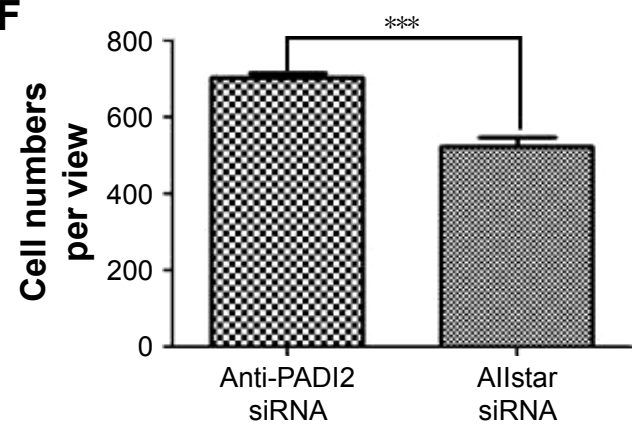

Figure 6 Migration of MNK-45 and Bel-7402 cells treated with anti-PADI2 siRNA, as measured by the transwell migration assay.

Notes: (A) MNK-45 cells were treated with anti-PADI2 siRNA. (B) MNK-45 cells treated with Allstar siRNA were used as negative controls. (C) Result of the migration measurement for MNK-45 cells. (D) Bel-7402 cells were treated with anti-PADI2 siRNA. (E) Bel-7402 cells treated with Allstar siRNA were used as negative controls. (F) Result of the migration measurement for Bel-7402 cells. Original magnification: $4.2 \times$. $* * * P<0.001$.

Abbreviation: PADI2, peptidylarginine deiminase isoform 2.

treatment with anti-PADI2 siRNA, a finding that corresponds to the result of PCR array. This study did not detect a significant change in the expression levels of ACSL5, AXIN2, CA9, CCL5, ESR1, IGFBP3, HMOX1, MMP2, MMP7 and TP63 in the siRNA-treated MNK-45 cells and Bel-7402 cells, or the expression profiles are different with the PCR assay. These results are shown in Figure 8.

\section{Discussion}

In the present study, immunohistochemistry detected the obvious expression of PADI2 in most tissue samples from breast-invasive ductal carcinoma, cervical squamous cell carcinoma, colon adenocarcinoma, esophageal tissues, gastric tissues, liver hepatocellular carcinomas, lung cancer, ovarian serous papillary adenocarcinoma, rectal tissues and papillary thyroid carcinoma samples. Immunoreactive score analysis indicated significantly increased expression of PADI2 in breast-invasive ductal carcinoma, cervical squamous cell carcinoma, colon adenocarcinoma, liver hepatocellular carcinomas, lung cancer, ovarian serous papillary adenocarcinoma and papillary thyroid carcinoma samples. In addition, our ELISA detected a significant increase in the PADI2 level in the blood samples of patients with liver cancer, cervical cancer and gastric carcinoma that correlated with the immunochemical results. Together with the current immunohistochemical observation, immunoreactive score analysis and ELISA measurement, we demonstrate that PADI2 shows high expression in many tumor tissues and in the blood of the patients, particularly in those with breast cancer, cervical carcinoma, liver cancer or gastric cancer. In our recent study, we used the Sequenom MassARRAY to determine the potential association of the PADI2-encoding gene with various tumor risks. Our study indicated that the single nucleotide polymorphisms rs2746533, rs2076616 and rs10788656 show significant differences in allele frequency, genotype frequency or both between breast cancer, cervical carcinoma, gastric carcinoma, lung cancer and rectal carcinoma cases and the controls, ${ }^{10}$ indicating that PADI2 has genetic susceptibility to these tumor risks. These findings suggest that the PADI2 might be related to the tumorigenesis of many types of tumors. Based on our immunohistochemistry and genotyping results, we continued to investigate the pathogenic pathway of PADI2 in gastric cancer and liver cancer. We have published our investigation of the expression and tumorigenic mechanism of PADI2 in MCF-7 cells originating from breast tumor tissues. ${ }^{10}$

The tumorigenic pathway of PADI2 was analyzed in MNK-45 and Bel-7402 tumor cell lines, two tumor cell models from gastric cancer and liver cancer, using tumorigenesisrelated PCR arrays. The siRNA interference on PADI2 expression resulted in the significantly altered expression of selected 


\section{A Significantly alternative gene \\ expressions detected by \\ Cancer PathwayFinder Pathway}
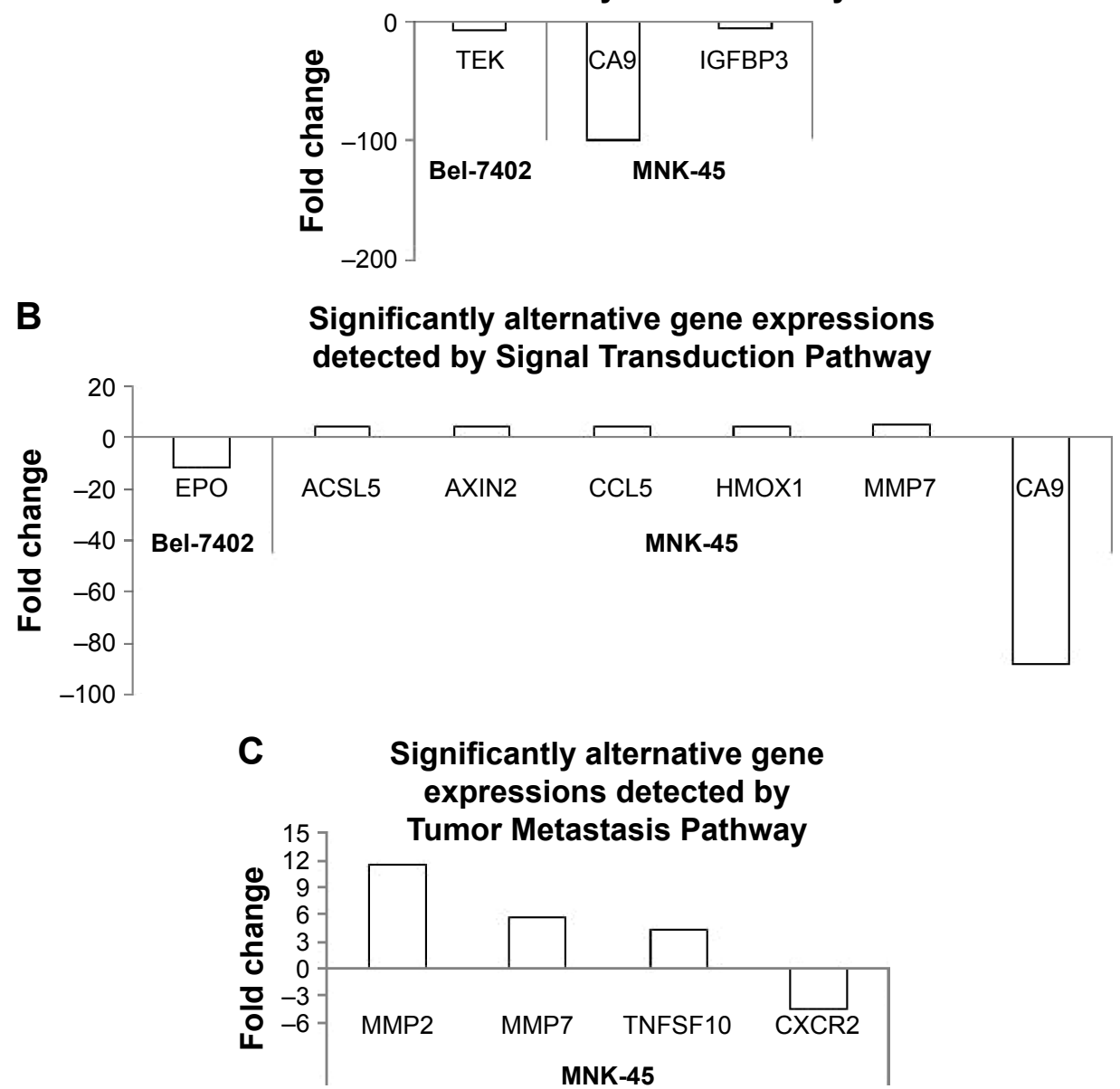

Dignificantly alternative gene
expressions detected by oncogenes
and Tumor Suppressor Genes Pathway

E Significantly alternative gene expressions detected by

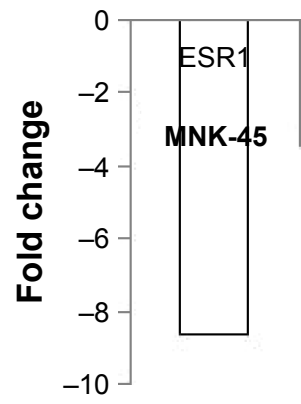
p53 Signaling Pathway

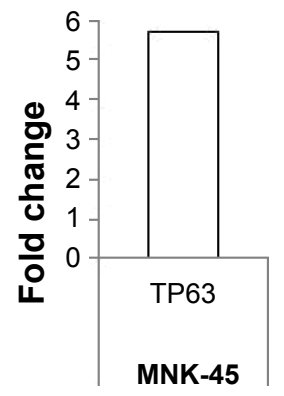

Figure 7 Determination of significantly altered gene expression using PCR arrays.

Notes: Tumor cell lines MNK-45 and Bel-7402 were treated with anti-PADI2 siRNA. Cells treated with Allstar siRNA were used as a negative control. Significantly altered expression of tumor-related genes was detected using (A) Cancer PathwayFinder PCR array, (B) Signal Transduction PCR array, (C) Tumor Metastasis PCR array, (D) Oncogenes and Tumor Suppressor Genes PCR array and (E) P53 Signaling PCR array.

Abbreviations: PCR, polymerase chain reaction; PADI2, peptidylarginine deiminase isoform 2.

genes involved in the tumorigenic progress, tumor suppression, p53 signaling, signal transduction and tumor metastasis. Finally, real-time PCR verified the decreased CXCR2 expression in the gastric tumor cells and decreased EPO expression in the liver cancer cells when PADI2 expression was suppressed.
The finding indicates that PADI2 might exert its tumorigenic function by mediating the expression levels of CXCR2 and $\mathrm{EPO}$ in gastric tumor and liver tumor cells, respectively.

C-X-C motif ligand 8 (CXCL8) is a well-known important multifunctional cytokine that promotes tumor proliferation, 

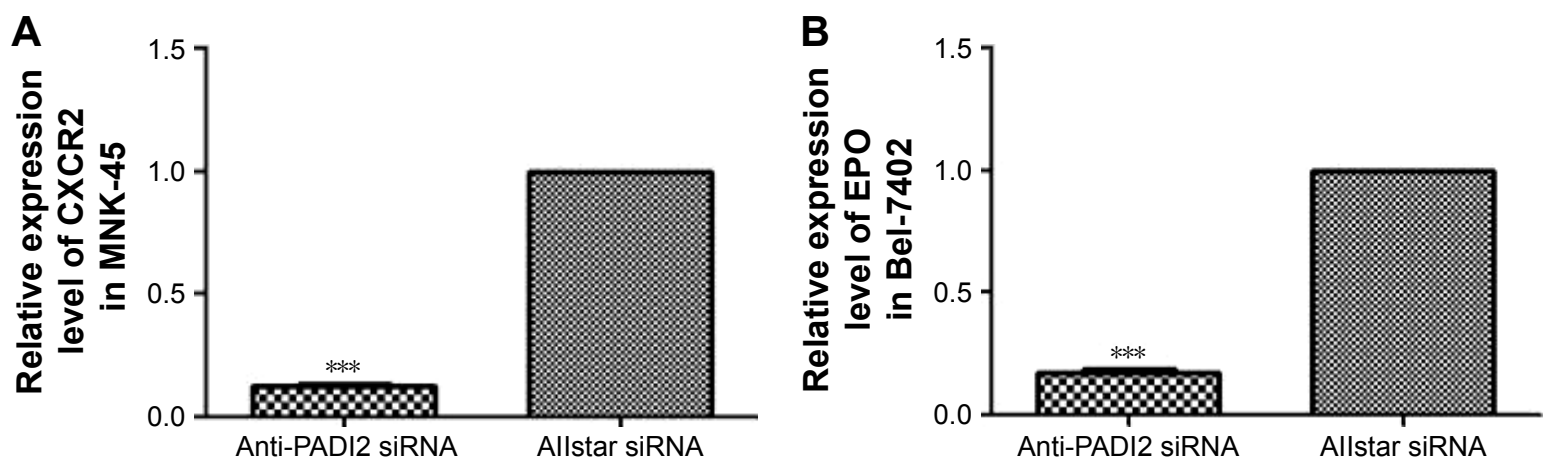

Figure 8 Determination of the mRNA expression levels of CXCR2 and EPO using real-time PCR.

Notes: MNK-45 cells and Bel-7402 cells were treated with anti-PADI2 siRNA. The transcription levels of the target genes in Allstar siRNA-treated cells were set as I. The target genes in the anti-PADI2 siRNA-treated cells were normalized to their expression in the controls. (A) CXCR2 expression level in MNK-45 cells and (B) EPO expression level in Bel-7402 cells. $* * * P<0.001$.

Abbreviations: PCR, polymerase chain reaction; PADI2, peptidylarginine deiminase isoform 2.

invasion and migration in an autocrine or paracrine manner. CXCL8 takes a function with its cognate receptors C-X-C chemokine receptor 1 (CXCR1) and CXCR2. ${ }^{14,15} \mathrm{CXCL} 1$ and CXCR2 show increased expression in gastric cancer tissues compared with adjacent noncancerous tissues, and CXCL1 or CXCR2 depletion significantly reduced the migration and invasion ability. ${ }^{16}$ Repertaxin, an inhibitor of the chemokine receptors CXCR1 and CXCR2, can inhibit the malignant behavior of human gastric cancer MNK-45 cells in vitro and in vivo. ${ }^{17}$ These reports are similar to our observation of decreased cell proliferation and cell migration in antiPADI2-treated MNK-45 cells while CXCR2 expression was depressed. Thus, we suggest that PADI2 contributes to cell migration and invasion in gastric tumors by upregulating CXCR2 expression.

Many tumors express EPO and/or the EPO receptor. Certain studies reported that various tumors express EPO to improve vascularization, thus favoring the growth and spread of tumor. EPO/EPO receptor expression is correlated with vascular mimicry formation. Patients with EPO or EPO receptor expression exhibited poorer overall survival than EPO-negative or EPO receptor-negative patients. ${ }^{18}$ It has also been reported that EPO promotes the proliferation and survival of cancer cells that express the EPO receptor. ${ }^{19,20}$ EPO is expressed in hepatocellular carcinoma cells and the human hepatoma cell line Hep3B..$^{21,22}$ However, evidence supporting a tumor growth-promoting effect of EPO has been inconclusive. Ehnert et $\mathrm{al}^{23}$ showed that darbepoetin, an EPO derivative, reduces the growth of Huh 7 cells, a hepatocarcinoma cell line, through the upregulation of the tumor-suppressor gene p53. Thus, EPO could delay tumor growth as several preclinical studies have reported. These reports correspond to our observation in which increased cell proliferation and cell migration were detected in anti-PADI2
siRNA-treated Bel-7402 cells with inhibited EPO expression. The present study offers evidence that PADI2 might have deleterious effects on tumor growth and metastasis in liver cancers by upregulating EPO expression. Cantarino et $\mathrm{al}^{11}$ demonstrated that the downregulation of PADI2 is an early event in colorectal carcinogenesis, a finding that corresponds to our observation in Bel-7402 cells with suppressed PADI2 expression.

The present study shows decreased cell proliferation and cell migration and increased apoptosis in anti-PADI2 siRNA MNK-45 cells with decreased CXCR2 expression. However, this study produced a controversial observation in the treated Bel-7402 cells with increased cell proliferation, cell migration and decreased apoptosis, whereas EPO expression was suppressed. We previously found that inhibiting PADI2 expression significantly increased the expression of CA9 and decreased the expression of ACSL4 and BIRC3 in MCF-7 cells, originating from breast cancer. Inhibiting PADI2 expression also significantly decreased the cell migration ability of MCF-7 cells but did not affect cell proliferation and apoptosis. ${ }^{10}$ The observations demonstrate that PADI2 has different effects and different tumorigenic pathways in different tumor cells. It is possible that the effect of PADI2 is multifactorial depending on the tumor type. We recently reported that PADI4, a PAD member, has genetic susceptibility to gastric carcinoma and upregulates CXCR2, KRT14 and TNF- $\alpha$ expression levels in MNK-45 cells. ${ }^{24}$ This result suggests that both PADI2 and PADI4 play a role via a similar signaling pathway in gastric tumorigenesis.

\section{Conclusion}

In summary, the present study showed significantly increased expression of PADI2 in many tumor tissues and in the blood of patients with cancer. Moreover, the study found that 
PADI2 at a low level can decrease cell proliferation and migration and advance apoptosis in gastric cancers with declined CXCR2 expression. In addition, suppressed PADI2 expression can promote cell proliferation and migration in liver cancer cells by downregulating EPO expression. The results suggest that the effect of PADI2 on tumorigenesis is multifactorial, depending on the tumor type. These findings might be useful for further understanding the tumorigenic process in gastric tumors and liver tumors.

\section{Acknowledgments}

This study was supported by the National Natural Science Foundation of China (81373218), Shandong Science and Technology Promotion Program (2015GGH318019, 2014GSF118062 and ZR2014HL045) and Projects of Medical and Health Technology Development Program of Shandong Province (2014WS0021).

\section{Disclosure}

The authors report no conflicts of interest in this work.

\section{References}

1. Pritzker LB, Nguyen TA, Moscarello MA. The developmental expression and activity of peptidylarginine deiminase in the mouse. Neurosci Lett. 1999;266(3):161-164.

2. Arita K, Hashimoto H, Shimizu T, Nakashima K, Yamada M, Sato M. Structural basis for $\mathrm{Ca}(2+)$-induced activation of human PAD4. Nat Struct Mol Biol. 2004;11(8):777-783.

3. Chavanas S, Méchin MC, Nachat R, et al. Peptidylarginine deiminases and deimination in biology and pathology: relevance to skin homeostasis. J Dermatol Sci. 2006;44(2):63-72.

4. Mohanan S, Cherrington BD, Horibata S, McElwee JL, Thompson PR, Coonrod SA. Potential role of peptidylarginine deiminase enzymes and protein citrullination in cancer pathogenesis. Biochem Res Int. 2012; 2012:895343.

5. Méchin MC, Coudane F, Adoue V, et al. Deimination is regulated at multiple levels including auto-deimination of peptidylarginine deiminases. Cell Mol Life Sci. 2010;67(9):1491-1503.

6. Lewis HD, Nacht M. iPAD or PADi-'tablets' with therapeutic disease potential? Curr Opin Chem Biol. 2016;33:169-178.

7. McElwee JL, Mohanan S, Griffith OL, et al. Identification of PADI2 as a potential breast cancer biomarker and therapeutic target. BMC Cancer. 2012;12:500.
8. McElwee JL, Mohanan S, Horibata S, et al. PAD2 overexpression in transgenic mice promotes spontaneous skin neoplasia. Cancer Res. 2014; 74(21):6306-6317.

9. Tanday S. Targeting PADI2 could stop the progression of myeloma. Lancet Oncol. 2016;17(8):e325.

10. Wang H, Xu B, Zhang X, Zheng Y, Zhao Y, Chang X. PADI2 gene confers susceptibility to breast cancer and plays tumorigenic role via ACSL4, BINC3 And CA9 signaling. Cancer Cell Int. 2016;16:61.

11. Cantarino N, Musulen E, Valero V, et al. Downregulation of the deiminase PADI2 is an early event in colorectal carcinogenesis and indicates poor prognosis. Mol Cancer Res. 2016;14(9):841-848.

12. Koo CL, Kok LF, Lee MY, et al. Scoring mechanisms of p16INK4a immunohistochemistry based on either independent nucleic stain or mixed cytoplasmic with nucleic expression can significantly signal to distinguish between endocervical and endometrial adenocarcinomas in a tissue microarray study. J Transl Med. 2009;7:25.

13. Briggs DJ, Skeeles JK. An enzyme-linked immunosorbent assay for detecting antibodies to Pasteurella multocida in chickens. Avian Dis. 1984;28(1):208-215.

14. Gales D, Clark C, Manne U, Samuel T. The chemokine CXCL8 in carcinogenesis and drug response. ISRN Oncol. 2013;2013:859154.

15. Liu Q, Li A, Tian Y, et al. The CXCL8-CXCR1/2 pathways in cancer. Cytokine Growth Factor Rev. 2016;31:61-71.

16. Cheng WL, Wang CS, Huang YH, Tsai MM, Liang Y, Lin KH. Overexpression of CXCL1 and its receptor CXCR2 promote tumor invasion in gastric cancer. Ann Oncol. 2011;22(10):2267-2276.

17. Wang J, Hu W, Wang $\mathrm{K}$, et al. Repertaxin, an inhibitor of the chemokine receptors CXCR1 and CXCR2, inhibits malignant behavior of human gastric cancer MKN45 cells in vitro and in vivo and enhances efficacy of 5-fluorouracil. Int J Oncol. 2016;48(4):1341-1352.

18. Yang Z, Sun B, Zhao X, et al. Erythropoietin and erythropoietin receptor in hepatocellular carcinoma: correlation with vasculogenic mimicry and poor prognosis. Int J Clin Exp Pathol. 2015;8(4):4033-4043.

19. Lombardero M, Kovacs K, Scheithauer BW. Erythropoietin: a hormone with multiple functions. Pathobiology. 2011;78(1):41-53.

20. Farrell F, Lee A. The erythropoietin receptor and its expression in tumor cells and other tissues. Oncologist. 2004;9(suppl 5):18-30.

21. Moreira L, Beirão JM, Beirão I, Pinho e Costa P. Oligomeric TTR V30M aggregates compromise cell viability, erythropoietin gene expression and promoter activity in the human hepatoma cell line Hep3B. Amyloid. 2015;22(2):93-99.

22. Li T, Liu Z, Jiang K, Ruan Q. Angiopoietin2 enhances doxorubin resistance in HepG2 cells by upregulating survivin and Ref-1 via MSK1 activation. Cancer Lett. 2013;337(2):276-284.

23. Ehnert S, Freude T, Eicher C, et al. Darbepoetin inhibits proliferation of hepatic cancer cells in the presence of TGF- $\beta$. Arch Toxicol. 2014; 88(1):89-96.

24. Zheng Y, Zhao G, Xu B, et al. PADI4 has genetic susceptibility to gastric carcinoma and upregulates CXCR2, KRT14 and TNF- $\alpha$ expression levels. Oncotarget. 2016;7(38):62159-62176.
OncoTargets and Therapy

\section{Publish your work in this journal}

OncoTargets and Therapy is an international, peer-reviewed, open access journal focusing on the pathological basis of all cancers, potential targets for therapy and treatment protocols employed to improve the management of cancer patients. The journal also focuses on the impact of management programs and new therapeutic agents and protocols on

\section{Dovepress}

patient perspectives such as quality of life, adherence and satisfaction. The manuscript management system is completely online and includes a very quick and fair peer-review system, which is all easy to use. Visit http://www.dovepress.com/testimonials.php to read real quotes from published authors. 